\title{
Les infirmières en oncologie ont-elles le pouvoir de créer
} leur propre avenir?

Par Rose Steele, inf., Ph.D.

Rose Steele, Ph.D., est professeure agrégée à l'École des sciences infirmières de l'Université York, à Toronto, en Ontario. Elle a également été rédactrice en chef de la Revue canadienne de soins infirmiers en oncologie de 1999 à 2004. Cet article est le texte du discours-programme qu'elle a présenté le mardi 28 septembre dans le cadre de la $16^{e}$ Conférence annuelle de l'ACIO à Calgary, Alberta.

Nous publions ce discours à la demande de participantes à la Conférence, qui ont exprimé le souhait de partager les idées de Mme Steele, Ph.D., avec le lectorat de la Revue.

Bonjour à toutes et à tous. Je suis très heureuse d'être parmi vous aujourd'hui, dans le cadre de cette Conférence annuelle de l'ACIO, dans la jolie ville de Calgary. Je suis particulièrement touchée d'être la conférencière d'honneur à l'occasion du 20e anniversaire de l'ACIO, et je vous remercie donc de m'avoir invitée. Vous savez, ce n'est pas facile de suivre la voie tracée par les conférencières d'honneur du passé. Je n'ai pas un sens musical très développé et je n'utiliserai guère d'analogies musicales dans mon discours, mais j'espère tout de même trouver mon propre rythme et ne pas trop vous ennuyer. Bien que je n'aie pas escaladé de vraie montagne, comme l'a fait Toben Anderson, j'ai dû, comme nombre d'entre vous, j'en suis certaine, escalader mes propres sommets imaginés. Et, tout comme Toben, je crois fermement que chacune d'entre nous a les moyens de devenir la personne qu'elle souhaite être et de créer sa propre vie, et c'est de cela que je vais vous parler aujourd'hui - mes idées au sujet de l'évolution des soins infirmiers, de leur situation actuelle et de la manière dont nous pouvons vivre le moment présent de façon à créer un avenir prometteur.

Certaines d'entre vous me connaissent peut-être par mon travail au sein de l'ACIO et particulièrement en tant que rédactrice en chef de la Revue canadienne de soins infirmiers en oncologie, la revue trimestrielle de l'ACIO évaluée par les pairs. Mais aujourd'hui, je vais vous en dire un peu plus à mon sujet afin de vous donner une idée de la raison pour laquelle j'éprouve une grande passion pour le rôle que chacune d'entre nous peut jouer dans sa propre destinée et dans le développement de notre profession. J'aimerais vous inviter à célébrer notre situation actuelle en tant qu'infirmières en oncologie, mais j'aimerais également vous lancer le défi de contribuer au façonnement de l'avenir des soins infirmiers en oncologie. Parce que, si nous ne le faisons pas nous-mêmes, quelqu'un d'autre s'en chargera pour nous

Voilà 30 ans maintenant que je travaille dans le domaine des soins infirmiers. J'ai fait mes débuts, en 1974, en tant qu'étudiante dans un programme en milieu hospitalier en Écosse, et j'ai obtenu mon diplôme d'infirmière après trois années complètes de dur labeur. Au fil des années, j'ai poursuivi mon éducation, à la fois en Écosse et ici au Canada, où je me suis installée en 1980. Je suis retournée aux études en 1986 afin d'obtenir mon B.Sc.inf. à l'Université de Toronto, parce que je voulais enseigner les sciences infirmières. J'avais été préceptrice d'élèves dans le milieu des soins intensifs où je travaillais et $\mathrm{j}$ 'aimais tout particulièrement ce volet des sciences infirmières. Mais je me suis aperçue que le B.Sc.inf. n'était que le début de mon périple. Le vaste monde des sciences infirmières m'est apparu d'une façon que je n'aurais jamais crue possible. Plus je perfectionnais mon éducation, plus je m'apercevais du peu que je savais vraiment, et cela m'a rendue très humble par rapport à ce que cette profession peut offrir - à l'infirmière individuelle et à la société en général. Je crois que les soins infirmiers peuvent jouer un rôle majeur dans le système des soins de santé canadien, mais sur de nombreux plans, les soins infirmiers n'ont jamais atteint leur plein potentiel.

Mon propre périple dans le milieu des soins infirmiers a été long et parsemé d'obstacles intéressants, mais je ne voudrais jamais abandonner cette carrière. Pourtant, je me sens frustrée du manque de progrès au sein de la profession. Lorsque je parle à des étudiantes du programme de premier cycle dans lequel j'enseigne, je m'aperçois que nombre d'enjeux auxquels elles font face aujourd'hui sont les mêmes que ceux auxquels nous nous heurtions il y a vingt ou trente ans. Comment cela est-il possible, et que pouvons-nous faire pour y remédier? J'aimerais vous raconter deux histoires: la première remonte à ma formation initiale en soins infirmiers, et la seconde date de plus tôt cette année. Ces histoires me serviront de toile de fond pour ma présentation d'aujourd'hui, à mesure que je retrace, avec vous, certaines des raisons pour lesquelles les soins infirmiers exercent une influence relativement faible au sein du système de santé. Toutefois, je ne vous laisserai pas dans cette même situation de faiblesse. Je vais plutôt vous offrir des idées qui vous permettront de vous prévaloir du pouvoir et de l'influence qui sont à notre portée, de façon à ce que nous, les infirmières en oncologie, puissions façonner notre propre avenir plutôt que de voir d'autres nous en imposer un.

C'était le milieu de l'année 1977 et j'achevais mon programme de premier cycle, à Glasgow, en Écosse. J'avais presque 21 ans et je prévoyais débuter le programme de sage-femme à l'hôpital Guy's, à Londres, en Angleterre, l'année suivante. J'avais envoyé ma demande d'inscription et je m'attendais à être admise parce que j'étais l'une des meilleures étudiantes dans mon programme. J'ai donc été estomaquée lorsque j'ai reçu une lettre m'annonçant que ma demande avait été rejetée. Toutes sortes d'idées vous viennent à l'esprit dans un moment pareil, mais je me suis rapidement arrêtée sur la raison la plus probable du refus - ma lettre de référence, rédigée par la directrice des sciences infirmières, n'était pas encourageante.

J'avais été une excellente étudiante en classe et dans les salles, mais je n'étais pas l'étudiante typique qui s'empressait de se lever lorsqu'un médecin faisait son entrée dans la salle, afin de lui céder mon siège, le sourire aux lèvres. J'ai toujours eu un sens aigu de l'équité et de l'égalité. Nombre des attentes de notre profession ne me revenaient pas. Je questionnais le statu quo et je m'exprimais lorsque je ne n'étais pas d'accord avec quelque chose. La devise de l'école d'infirmières, que nous a citée Pamela Baker hier - "Je vois et je ne dis rien" - ne me convenait pas du tout alors et ne me convient toujours pas aujourd'hui. À deux reprises, lors de mes quarts en salle d'urgence, je m'étais plainte lorsque, selon moi, le manque de soins appropriés avait mené à la mort de patients, dont l'une n'avait que 16 ans et était décédée 
d'un état de mal épileptique traité de manière inadéquate. Je soupçonnais que ces plaintes revenaient alors pour me hanter.

J'ai fixé rendez-vous avec ma directrice. J'avais peur de la rencontrer, mais mon besoin d'équité m'a poussée à aller lui parler. Je n'arrivais pas vraiment à croire qu'on me punissait parce que je m'étais exprimée et je voulais en avoir le cœur net. La directrice s'est exprimée très clairement lorsque je lui ai posé la question. Elle n'avait pas écrit une bonne référence pour moi parce que j'avais besoin d'une leçon. Je n'étais qu'une infirmière, et une étudiante en plus. De quel droit osais-je m'exprimer contre ceux qui étaient "meilleurs" et plus puissants que moi - les médecins et les infirmières plus expérimentées? Puisque j'avais certainement appris ma leçon en n'étant pas admise à Guy's, elle m'écrirait maintenant la lettre de référence que je méritais pour que je puisse étudier dans toute autre école de sage-femme de mon choix.

Ceux et celles qui me connaissent savent que j'ai bel et bien appris une leçon ce jour-là. Mais ce n'était pas celle que la directrice voulait que j'apprenne. Ce que j'ai appris, c'est que nous sommes entourés de sources de pouvoir et que certaines personnes abusent de leur pouvoir. J'ai compris que je devais définir et cultiver mon propre pouvoir si je voulais réussir. Mais je devais également m'assurer de l'utiliser correctement. Nombre d'infirmières ne se rendent pas compte de la façon dont le pouvoir est utilisé dans le système de santé et ne savent pas non plus mettre à profit leur propre pouvoir. La prochaine histoire que je veux partager avec vous illustre la façon dont une nouvelle génération d'infirmières éprouve encore de nos jours un sentiment d'impuissance, alors que nous devons aller de l'avant si nous souhaitons que notre profession réalise son plein potentiel.

L'hiver dernier, j'enseignais un cours de préparation à la profession à des élèves de troisième année d'université. Le cours se donnait de $11 \mathrm{~h} 30$ à $14 \mathrm{~h} 30$, avec une pause de dix minutes pour chaque heure de cours; nous commencions donc habituellement à 11h30, nous prenions une pause de 15 minutes au milieu du cours, et nous terminions à $14 \mathrm{~h} 15$. Le sujet de la semaine était l'oppression et comme d'habitude, nombre d'étudiantes ne s'étaient pas donné la peine de faire les lectures hebdomadaires assignées. Je m'en suis aperçue lorsque j'ai vu leur réaction face aux renseignements que j'avais écrits au tableau:

\section{CHANGEMENTS À L'HORAIRE DE COURS (en vigueur dès maintenant)}

- Vu le volume important de matière à couvrir chaque semaine, j'ai décidé que nous n'avons pas assez de temps pour prendre une pause. - Par conséquent, aujourd'hui, nous travaillerons sans arrêt jusqu'à la fin du cours.

- Nous allons également prolonger le cours de 15 minutes, de façon à terminer à $14 \mathrm{~h} 30$.

- De plus, à partir de la 7e semaine (c.à-d. après la semaine de lecture), je m'attends à ce que vous arriviez à $11 \mathrm{~h} 20$, prêtes à commencer le cours.

- J'espère que ces mesures nous permettront d'accomplir une bonne partie du travail. Si je m'aperçois que nous n'avançons toujours pas assez, alors je réexaminerai l'horaire et prolongerai possiblement le cours jusqu'à 14 h45.

Bien entendu, je n'avais aucune intention de modifier l'horaire. En outre, je n'en avais pas le droit, selon les règlements de l'université. Je voulais plutôt utiliser le cadre de la relation professeure-étudiants pour discuter du thème de l'oppression au sein de la profession. Je voulais également faire le lien entre ce que j'imposais et ce que l'on tient pour acquis dans le milieu de travail. La grande majorité des étudiantes ont lu l'information au tableau et ont quitté la salle de classe immédiatement. Nombre d'entre elles étaient bouleversées, irritées, fâchées même, mais elles n'étaient pas capables ou n'avaient pas envie de faire face au fait que je semblais leur imposer des changements d'horaire. Les étudiantes qui sont restées ont pris part à un débat à la fois stimulant et fascinant, et j'ai envoyé un courriel aux autres étudiantes pour leur expliquer l'intention de cette stratégie pédagogique et pour leur donner un bref aperçu de ce qu'elles avaient manqué.

Si vous y réfléchissez bien, vous verrez probablement le parallèle entre ce que j'avais écrit au tableau et ce qui se passe dans l'environnement clinique. Dans beaucoup de milieux, on attend des infirmières qu'elles se présentent au travail 15 minutes à l'avance, qu'elles ne prennent aucune pause et qu'elles restent 30 minutes de plus après le travail pour terminer de rédiger leurs notes. Pourquoi cela est-il normal et que pouvons-nous faire pour changer la situation? Ces questions ont servi de point de départ à d'excellentes discussions en classe et constituent le fondement du reste de cette présentation. Prenons courage, car nous POUVONS changer la situation présente et nous POUVONS apprendre à effectuer ces changements.

\section{Contexte}

Il est important d'examiner notre contexte de travail puisque, même si les individus sont en mesure d'amener un changement une fois qu'ils savent comment s'y prendre, ils doivent également être conscients des contraintes de leur milieu et s'y adapter. Par exemple, j'étais une étudiante en sciences infirmières au sein d'un système qui méprisait les élèves et les infirmières en général. J'ai dû prendre conscience des contraintes qui découlaient de cette mentalité afin d'apprendre à travailler avec elles et à les contourner de façon à accomplir ce qui me semblait important. Il serait naïf d'imaginer que nous pouvons apprendre à exercer notre influence et notre pouvoir sans comprendre notre contexte. Toutefois, cela ne signifie pas qu'en fournissant les efforts appropriés, le contexte ne puisse pas être modifié lui aussi, en autant que les circonstances s'y prêtent et à force d'efforts. Le contexte est souvent façonné par des attentes de la société; ces attentes peuvent évoluer, mais cette évolution est souvent très lente. Certains des principaux facteurs d'influence qui sévissent dans nos milieux de travail et que l'on doit prendre en compte et gérer comprennent: 1) le manque de clarté concernant les tâches des infirmières, ce qui signifie que nous avons de la difficulté à fournir des preuves quant à l'incidence des soins que nous prodiguons; 2) des charges de travail de plus en plus lourdes à mesure qu'augmentent l'intensité et la complexité des soins aux patients; 3) un manque de contrôle sur la façon dont nous nous acquittons de nos tâches et sur le moment où nous nous en acquittons; 4) une incohérence fréquente entre l" "idéal" auquel nous aspirons et la "réalité" dans laquelle nous vivons, ce qui induit un stress et une angoisse chez bon nombre d'infirmières; 5) un manque de soutien et de ressources.

\section{Le facteur genre}

Une autre influence majeure sur la vie professionnelle des infirmières et sur notre capacité de créer notre avenir nous-mêmes est le genre. Ce terme reflète la façon dont une personne vit sa vie par rapport à la masculinité et de la féminité. Le genre n'est pas synonyme de "sexe" et n'est donc pas un rapport déterminé par la biologie. Il s'agit plutôt d'une construction sociale et on le tient généralement pour acquis, bien qu'il soit souvent mal compris. Le genre s'accompagne de valeurs culturelles et, en général, les gens se comportent de façon à renforcer les constructions dominantes de la société. De fait, de nombreuses personnes acceptent sans les questionner les définitions et attentes sociétales relatives aux rôles assignés aux genres, et les membres d'une société contribuent ainsi à maintenir l'ordre "normal" des idées. Les enfants apprennent dès un jeune âge ce que signifient la masculinité et la féminité dans leur société et ils apprennent rapidement à se conformer aux attentes sociales qui correspondent à leurs genre et sexe. Toutefois, les définitions et attentes en vigueur peuvent évoluer dans le temps et l'espace; un examen des rôles assignés aux genres peut donc mener à des changements dans ce que l'on considère "normal" au sein d'une société (McDonald, 2003). 
La notion de genre a une incidence sur les infirmières au niveau individuel et institutionnel. Elle influence la valeur qui est accordée à notre savoir et à notre travail au sein de la société et mène à des divisions professionnelles (ou horizontales) fondées sur le genre. Ainsi, certaines professions sont jugées appropriées pour les hommes et non pour les femmes, et vice versa. Le genre mène également à des divisions hiérarchiques (ou verticales) fondées sur le genre; ainsi, les professions dites "masculines" sont mieux rémunérées et sont sources de davantage de pouvoir et de prestige que les professions dites "féminines". De plus, les attentes par rapport au rôle des femmes en tant que "dispensatrices de soins" au sein des relations personnelles et familiales ont une incidence sur la capacité des femmes à gérer les responsabilités et les demandes de temps qui vont souvent de pair avec les rôles plus "élevés" tels que les postes de cadres (McDonald, 2003). Est-il donc étonnant de voir un nombre disproportionné d'hommes occuper les postes de direction en soins infirmiers (RossKerr, 2002)?

Le genre joue un rôle dans les soins infirmiers parce que le métier est associé à certaines vertus dites "féminines", telles que la bienveillance, ce qui a une incidence sur les deux sexes. Les caractéristiques associées à la féminité comprennent entre autres les suivantes: un caractère soumis, l'impuissance, le manque d'autonomie, la tendresse, la tendance à se dévouer et l'altruisme. À la masculinité, on associe davantage la force, l'agression, la maîtrise, l'indépendance, la logique, la réflexion non émotive, la compétitivité et l'ambition. Mais ces traits n'appartiennent-ils vraiment qu'à l'un ou à l'autre des deux sexes? En tant que patient, préféreriez-vous être soignée par une personne qui manque d'autonomie, qui est impuissante mais sait se dévouer ou par une personne qui est forte, agressive et compétente? En tant qu'infirmière, vous voyez-vous comme "l'une ou l'autre" de ces deux personnes? Les grandes infirmières ne réunissent-elles pas les meilleurs traits de l'un et l'autre de ces ensembles de caractéristiques? Pourtant, lorsqu'on examine les soins infirmiers du point de vue de la féminité, la signification et l'importance du "travail de femme" se voient diminuées, et nos connaissances et façons d'être sont dévalorisées. De plus, les conditions sociales restreignent et guident les choix qui s'offrent aux femmes en ce qui est du travail et de la vie personnelle, avec pour résultat que les soins infirmiers demeurent une profession avant tout féminine, limitée par les contraintes qui vont de pair avec le fait d'être femme dans notre société (McDonald, 2003).

\section{L'oppression}

Le contexte de nos vies professionnelles en soins infirmiers a été comparé à la vie dans une société opprimée. Bien que toutes les infirmières n'aient peut-être pas déjà entendu cette analogie, je me demande combien d'entre vous se reconnaîtront ou reconnaîtront leurs collègues et/ou la profession des soins infirmiers en général dans certaines des caractéristiques d'un groupe opprimé que je me propose de vous présenter. À tout le moins, cela vous donnera peut-être matière à réflexion.

Les membres de groupes opprimés sont contrôlés par des forces extérieures qui jouissent de plus de prestige, de pouvoir et d'influence qu'eux, et ils sont exploités par un groupe plus puissant qu'eux. Cela ne vous rappelle-t-il pas la relation médecin-infirmière? Les groupes opprimés intériorisent les normes et valeurs du groupe dominant et en viennent à considérer ces normes comme "justes", même si c'est au prix du rejet de leurs propres caractéristiques dans une tentative de s'intégrer un tant soit peu, d'être davantage comme le groupe dominant. À mesure que les membres du groupe opprimé abandonnent leurs propres valeurs, ils en viennent à se détester euxmêmes et manifestent souvent des comportements passifs-agressifs. Cela mène fréquemment à des conflits au sein du groupe (violence horizontale), et l'on voit les membres s'attaquer mutuellement au lieu de s'en prendre à ceux de l'extérieur du groupe qui sont perçus comme plus puissants. Cette violence horizontale naît souvent de la peur. Peur que le groupe subordonné puisse être détruit s'il se révoltait et peur de changer le statu quo parce que cela entraînerait des changements si la révolution réussissait et que les gens seraient mal à l'aise face au changement (Roberts, 1983).

Le statu quo est maintenu de diverses façons. Les infirmières acquièrent des comportements jugés acceptables par le groupe dominant et elles craignent l'agression ou la rétorsion si elles outrepassent leurs limites. Cela n'est pas sans raisons, comme le démontre mon expérience d'étudiante. Bien que cela ne m'ait jamais empêchée de m'exprimer, j'ai parfois dû subir des conséquences négatives. Mais soyez certaines que le fait de m'exprimer a également mené à des changements constructifs dans diverses situations. Le leadership au sein du groupe opprimé a tendance à refléter la culture du groupe dominant, d'où le fait qu'une dirigeante au franc parler qui défend les droits de son personnel est généralement mal vue par le groupe dominant. Paradoxalement, il se peut que les membres du groupe dominé maltraitent également cette chef parce qu'ils ne croient pas qu'elle soit réellement "de leur côté". Cela crée une situation très inconfortable pour les dirigeants qui luttent contre le statu quo et qui font de leur mieux pour servir les personnes avec ils travaillent.

Certaines chefs de groupes démunis de pouvoir acquièrent des attitudes négatives envers leurs "pairs" à mesure qu'elles tentent de s'associer au groupe dominant. Ces chefs peuvent se voir décerner des récompenses, telles qu'une promotion, pour leurs "bons" comportements; des gestes symboliques tels que la remise de récompenses et la reconnaissance de droits mineurs contribuent à garder au minimum le niveau d'insatisfaction. Les mythes au sujet de la culture dominante qui circulent au sein du groupe opprimé sont encouragés et partagés avec les nouveaux membres de la profession, et le système de formation soutient souvent le statu quo en assurant la propagation des vieilles croyances (Roberts, 1983). Pour ne citer qu'un exemple, l'enseignement typique que reçoivent les médecins met l'accent sur leur rôle en tant que décideurs de la plus haute instance dans la pratique. La formation pluridisciplinaire est presque inexistante, si bien que les professionnels de la santé ne sont souvent pas conscients des compétences et des connaissances que leurs collègues peuvent apporter au processus décisionnel.

Il est possible de changer le statu quo en prenant conscience de l'oppression, en rejetant les mythes et en les remplaçant par des images positives de sa propre culture, en acquérant de l'autonomie et en faisant preuve de leadership à l'intérieur du groupe afin de développer un sentiment de cohésion et de fierté parmi les membres du groupe. Le continuum de développement d'une identité positive (Roberts, 2000) dont je vais maintenant vous parler peut vous aider à analyser votre situation présente et guider vos pas vers une destination plus enviable.

À l'extrême gauche du continuum se situe l'attitude de prérencontre ou d'acceptation non vérifiée, qui se caractérise par l'acceptation, de la part des infirmières, de leur statut opprimé, des rôles actuels des infirmières, des structures du pouvoir en place et du pouvoir des médecins. Les personnes qui se situent à cette extrémité ont tendance à avoir une attitude négative envers les soins infirmiers. Je me demande combien parmi vous ont déjà entendu ou prononcé une phrase du genre: "J'aime les soins infirmiers, mais je n'encouragerais jamais mon fils ou ma fille à devenir infirmier ou infirmière".

Un peu plus à droite sur le continuum se situe la rencontre ou la prise de conscience. Votre réalité subit une transformation, et vous reconnaissez l'injustice de votre situation actuelle. Il se peut que vous vous sentiez injustement traitée que vous ayez tendance à voir la situation comme toute blanche ou toute noire, de façon que seules les infirmières ont toujours raison. Lorsque vous avez besoin de soutien, vous avez tendance à le chercher auprès d'autres infirmières, mais cette attitude peut finir par vous isoler des autres professionnels de la santé. 
Avec le temps, l'immersion-émersion ou la connexion débouche sur un sentiment accru de fierté et sur une identité positive en tant qu'infirmière. Vous êtes alors plus susceptible de vous joindre à des groupes d'infirmières et d'échanger des idées avec d'autres infirmières au sujet des possibilités. L'avenir de la profession semble alors meilleur.

L'intériorisation ou la synthèse se caractérise par l'acquisition d'une nouvelle identité, et vous intériorisez des idées positives au sujet de la profession. Vous ne voyez pas votre situation en tant que toute blanche ou toute noire, en tant que tout ou rien; par exemple, au lieu de voir les médecins en tant que groupe uniforme, vous voyez des individus ainsi que leurs forces. Les relations interdisciplinaires deviennent alors importantes. Vous donnez la priorité à la résolution de problèmes de façon à faire la différence sur le plan des soins infirmiers et de votre vie professionnelle. Vous réalisez que les infirmières se distinguent des autres professionnels de la santé tout en étant leurs égaux.

Finalement, à l'extrême droite du continuum se situent l'engagement et l'action politique qui visent la justice et le changement sociaux. Cela peut vous amener à prendre part à des activités de promotion telles que la justice sociale. À mon avis, Cathy Crowe, de Toronto, est un excellent exemple d'une infirmière qui exerce son métier dans une optique de justice sociale et d'action politique.

Où vous situez-vous sur ce continuum? Où souhaiteriez-vous être? Selon vous, où se situe la profession des soins infirmiers? Si vous décidez d'aller de l'avant et d'amorcer un changement, comment pouvez-vous vous y prendre? Permettez-moi de vous donner deux indices: le pouvoir et l'influence.

\section{Le pouvoir}

Nombreuses sont les femmes qui n'aiment pas le mot pouvoir. Pourtant, il constitue une partie essentielle de la vie. Le pouvoir décrit la capacité d'exercer une influence, ce qui n'est pas la même chose que l'influence en soi, un concept que j'examinerai un peu plus loin. L'influence est l'utilisation du pouvoir, et il est essentiel que les infirmières reconnaissent et exercent leur pouvoir. L'autorité et le pouvoir sont deux concepts différents. Chaque interaction humaine est empreinte de pouvoir. Ce dernier peut se manifester de façon symétrique ou asymétrique, mais l'équilibre est préférable à la concentration du pouvoir chez une seule des parties. Nous devons accepter le pouvoir en tant que réalité positive et nécessaire, mais il n'est pas nécessaire de le poursuivre comme un but en soi. Le partage du pouvoir survient naturellement lorsque le pouvoir est centré sur les valeurs et les principes de chacun, et c'est ce genre de pouvoir qui vient naturellement à bien des femmes (Sullivan, 2004).

Le pouvoir fondé sur les principes est un concept qui s'intègre facilement aux valeurs des soins infirmiers. Il est basé sur le respect, l'honneur, la loyauté et l'engagement. Il se caractérise par: 1) la compréhension des autres et de leurs besoins et désirs; 2) le partage de renseignements avec autrui; 3) l'expression des idées de façon précise et enthousiaste; 4) l'utilisation des pouvoirs pour encourager autrui; 5) le respect des opinions différentes des siennes; 6) l'élargissement de son réseau de relations interpersonnelles. Bref, le pouvoir fondé sur les principes est un concept à la fois constructif et très positif.

\section{Types de pouvoir}

Les chercheurs ont cerné six types de pouvoir: 1) la position (autorité): découle du rôle que l'on occupe; 2) la possession de l'information: découle de l'accès aux renseignements; 3) l'expertise: découle du fait de posséder les connaissances spécialisées requises; 4) le pouvoir personnel: découle du respect ou de la crédibilité dont jouit une personne; 5) les relations: découle des liens entretenus avec des personnes d'influence; 6) la perception de pouvoir: découle du fait qu'autrui est persuadé que vous détenez du pouvoir (Sullivan, 2004).

\section{Utilisation du pouvoir}

Les gens qui détiennent du pouvoir ont la responsabilité de connaître les règles concernant l'emploi qu'ils peuvent en faire. Vous devriez exercer la plus petite quantité de pouvoir requise pour obtenir les résultats souhaités et utiliser un pouvoir approprié pour la situation. Il est également important d'apprendre quand ne pas exercer son pouvoir. Lorsque vous exercez votre pouvoir, concentrezvous sur l'enjeu et non sur la personne. Veillez à ce que vos demandes soient polies et jamais arrogantes et n'ayez recours à la coercition que lorsque d'autres méthodes se sont avérées inefficaces. Tenez-vous informées afin de conserver votre crédibilité lorsque vous exercez votre pouvoir d'experte, et comprenez qu'il se peut que vous deviez une faveur aux personnes au pouvoir desquelles vous faites appel (Sullivan, 2004).

Le pouvoir utilisé de façon inappropriée est un horrible gaspillage. Il y a gaspillage lorsque le pouvoir exercé ne convient pas à la situation. Les personnes qui sous-utilisent leur pouvoir, c.-à-d. qui ne l'exercent pas dans une situation dans laquelle elles devraient l'exercer, perdent leur crédibilité et sont souvent perçues comme incompétentes. Celles qui utilisent leur pouvoir de façon excessive et qui réagissent aux situations de façon exagérée finissent elles aussi par s'attirer des ennuis. La surutilisation du pouvoir sème la peur et l'hostilité chez autrui et étouffe la créativité et la capacité de résoudre des problèmes. Cela se traduit souvent par un manque de confiance et la diminution de la capacité de réaliser ses objectifs. Vous perdez votre pouvoir lorsque vous prenez constamment de mauvaises décisions ou lorsque vous vous montrez incapable de prendre des décisions (Sullivan, 2004).

\section{Reconnaissez votre pouvoir}

Vous reconnaissez probablement les divers types de pouvoir et pourriez sans doute nommer plusieurs personnes qui ont du pouvoir et l'utilisent bien ou l'exercent mal d'une façon ou d'une autre. Mais reconnaissez-vous votre propre pouvoir? Lui accordez-vous sa juste valeur, et réalisez-vous ce qui lui arrive lorsque vous ne l'exercez pas? Les gens qui n'utilisent pas leur pouvoir peuvent manquer des occasions qui leur seraient avantageuses ou qui seraient avantageuses pour leurs patients ou pour leur profession. Ces personnes peuvent sembler indécises et être perçues comme moins compétentes qu'elles ne le sont réellement. Cela risque de nuire à leur crédibilité, et leur efficacité future s'en verra diminuée. Finalement, il se peut que l'on écarte leur opinion et qu'elles soient ignorées dans nombre de situations (Sullivan, 2004).

À l'occasion du 20e anniversaire de l'ACIO cette année, les membres de l'ACIO ont eu l'occasion d'exercer leur pouvoir en partageant des récits au sujet des effets positifs qu'ils ont dans la vie d'autrui en tant qu'infirmières en oncologie. C'était une occasion de mettre notre profession en vedette et de vanter nos mérites haut et fort! Malheureusement, la Revue n'a pas reçu une seule réponse. Imaginez la sensation de pouvoir qui aurait accompagné le partage de ces récits avec d'autres professionnels de la santé ou tout simplement entre nous. C'est toute une occasion que nous avons manquée là!

\section{L'accroissement du pouvoir}

J'espère que vous commencez maintenant à penser que le fait de posséder du pouvoir n'est peut-être pas une si mauvaise chose et qu'au contraire, cela pourrait vous être avantageux. Donc, comment faire pour accroître votre pouvoir au sein d'un organisme de façon à changer le statu quo et à commencer à prendre en main votre propre avenir? Une façon est de vous rendre plus visible au sein de votre organisme de travail. Augmentez votre niveau d'engagement, assurez-vous de faire votre travail de façon efficace et efficiente et assumez de nouvelles responsabilités, selon vos capacités. Apprenez le plus possible au sujet de l'organisme et des gens qui en font partie. N'ayez pas peur de demander de l'aide ou des conseils et continuez 
de vous développer sur le plan personnel et d'acquérir de nouvelles compétences professionnelles. Vous devriez faire de l'apprentissage permanent un objectif indéfectible de votre vie professionnelle. Prenez position lorsqu'il y a un débat. Ne soyez pas un mouton qui se contente de suivre les autres. Au contraire, donnez votre opinion après avoir attentivement considéré les faits. Pesez le pour et le contre. Épousez le changement et cherchez les aspects positifs de la situation au lieu de vous contenter de garder vos distances et de tout critiquer. Je ne vous encourage pas à chercher le changement comme un but en soi. Mais je trouve dommage que nombre de changements qui ont beaucoup de potentiel ne voient pas le jour à cause du manque d'enthousiasme de personnes qui, tout en n'étant pas nécessairement opposées à l'idée du changement, ne veulent pas avoir à s'y adapter. N'oubliez pas d'établir des liens au sein de l'organisme et dans autant de milieux que possible. Assurez-vous de vous associer aux décideurs lorsque cela est approprié. Et finalement, partagez votre pouvoir avec autrui; plus vous le partagez, plus vous le cultivez (Sullivan, 2004).

\section{L'érosion du pouvoir}

Nous avons toutes et tous la capacité d'être puissants. Pourtant, bon nombre d'entre nous affaiblissons notre propre pouvoir (ou celui d'autrui) sans nécessairement nous en apercevoir. Notre crainte instinctive du changement nous retient. L'idée d'effectuer des changements ou d'avoir à nous y adapter nous effraie, si bien que nous trouvons des façons de prévenir le changement. Pourtant, si nous n'épousons pas le changement, comment pouvons-nous nous attendre à amener un changement dans le cadre de nos vies professionnelles? Je crois qu'une de mes définitions non médicales préférées est très apte dans ce contexte. Qui connaît la définition de la folie? La folie consiste à répéter sans cesse les mêmes comportements dans l'espoir d'obtenir un résultat différent. C'est tellement vrai! Pouvez-vous m'expliquer pourquoi nous devrions nous attendre à ce que quoi que ce soit change ou s'améliore si nous ne modifions pas notre comportement afin d'obtenir un résultat différent?

Je suis certaine que certaines d'entre vous se reconnaîtront ou reconnaîtront des collègues dans la liste suivante de comportements qui diminuent le pouvoir. Si tel est le cas, je ne veux pas que vous vous sentiez mal à l'aise ou bouleversées. Prenez plutôt la décision d'accepter votre pouvoir et de travailler à l'accroître tout en vous efforçant d'éliminer les comportements qui sabotent votre pouvoir. Un des comportements que j'aime le moins et que j'essaie d'afficher le moins souvent possible est la plainte stérile. Se plaindre ainsi, c'est émettre des commentaires négatifs au sujet de quelque chose ou de quelqu'un sans lui dire en face. Cela se produit lorsque vous parlez de votre chef dans son dos, par exemple, au lieu d'exprimer vos préoccupations d'une façon qui invite une discussion ouverte et une éventuelle solution. Je suis certaine que vous savez de quoi je parle. Les plaintes continues constituent un autre exemple de comportements qui ne mènent nulle part et peuvent vous donner mauvaise réputation. Au lieu d'inventer des excuses pour éviter d'essayer quelque chose, vous devez résoudre les difficultés en examinant des solutions de rechange. Soyez prêtes à prendre un risque, et même à échouer. D'autres comportements qui sapent le pouvoir sont les phrases qui tuent le changement dans l'œuf. En voici quelques exemples: On ne peut pas faire ça parce que... "Elle va se fâcher" ou "Ils vont penser que je suis vaniteuse" ou "Ça va coûter trop cher" ou "L'administration n'acceptera jamais ça", et ainsi de suite. Vous les reconnaissez? Avec quelle fréquence les utilisez-vous? $\mathrm{Ne}$ soyez pas négatives! Vous devez trouver des solutions et des façons de mettre en œuvre une idée si elle est prometteuse (Sullivan, 2004).

Les deux derniers comportements nocifs peuvent être difficiles à contrôler, mais leur maîtrise en vaut la peine. Il est très facile d'avoir une réaction émotionnelle à une suggestion d'autrui, mais afficher une telle réaction est susceptible d'être autodestructeur. Vous devez vous rendre compte que tout ce qui arrive dans le milieu de travail concerne le travail; vous ne devriez jamais vous sentir personnellement visées, même si cela est souvent difficile pour nous, les femmes. Prenez le temps de vous recentrer puis discutez de la question d'une façon plus professionnelle et rationnelle. N'oubliez pas qu'il est possible de débattre et même d'attaquer des idées, mais on ne devrait jamais attaquer les individus. Finalement, un trop grand nombre de personnes refusent d'admettre leurs erreurs, et cela mène à l'érosion de leur pouvoir. Si vous commettez une erreur, il est important d'en assumer la responsabilité et d'offrir de remédier à la situation. Mais il convient ensuite de passer à autre chose. Ne vous fustigez pas pour vos erreurs. Apprenez à faire la différence entre des erreurs mineures et majeures et prenez les mesures qui s'imposent (Sullivan, 2004). N'oubliez pas que de nombreuses découvertes ont été faites grâce à des erreurs .

\section{Allons de l'avant pour créer notre propre avenir en tant qu'infirmières en oncologie}

Jusqu'à présent, nous avons examiné certains des obstacles qui entravent notre capacité de créer notre propre avenir en tant qu'infirmières en oncologie et nous avons appris l'importance du pouvoir ainsi que les façons d'accroître notre pouvoir. Nous devons à présent examiner la façon dont nous pouvons utiliser notre pouvoir pour amener le changement et pour prendre notre avenir en main. Je veux prouver que nous avons du pouvoir et que nous pouvons nous en servir. Je vais donc illustrer cette idée en examinant trois comportements positifs, à savoir l'utilisation de l'influence, devenir chef de file et l'adhésion à des organismes professionnels.

\section{L'influence}

Le Canada compte près de 250000 infirmières qui pratiquent le métier, et nous représentons presque la moitié des travailleurs de la santé de notre système (Construire l'avenir, 2004). Imaginez l'influence que nous aurions si nous apprenions à canaliser et à exercer notre pouvoir! Donc, qu'est-ce que l'influence?

L'influence est la capacité de communiquer ses idées aux autres et de gagner leur soutien par le biais de l'acceptation ou de la participation. Elle ne peut exister que dans les relations avec autrui et est associée à un but précis dans le contexte des volets personnel et professionnel de votre vie. L'influence est plus importante que l'autorité et elle est déterminée par tous les aspects de votre personne, tant sur le plan personnel que professionnel. L'influence peut s'accorder ou se gagner et peut être vaste ou limitée. Il est possible d'enseigner et d'apprendre les aptitudes liées à l'influence, mais les événements du passé ont des répercussions sur la capacité d'exercer de l'influence (Sullivan, 2004). Avant d'examiner la façon dont vous, en tant qu'individus, pouvez acquérir de l'influence, il est important de retourner au contexte historique et de décrire les trois principales influences qui ont façonné la situation actuelle des soins infirmiers. Cela vous permettra de vous faire une idée des raisons pour lesquelles notre profession a toujours lutté pour avoir de l'influence.

\section{Influences historiques sur les soins infirmiers}

Florence Nightingale a été la première personne d'influence dans la profession des soins infirmiers. Si vous vous rappelez vos cours d'histoire, vous savez que le passé des soins infirmiers présente des liens étroits avec l'histoire militaire, en commençant par le travail de Florence durant la Guerre de Crimée. Le milieu militaire exige de la discipline, l'obéissance aux ordres et le respect de la chaîne de commandement, et les soins infirmiers ont également adopté ces caractéristiques. Notre passé affiche également des liens solides avec l'histoire religieuse, puisque les premiers hôpitaux et écoles d'infirmières ont été mis sur pied par des ordres religieux dans le but 
de venir en aide aux pauvres. Les ordres religieux accordaient beaucoup d'importance au service et à l'obéissance. Finalement, nous avons déjà abordé la dimension de l'histoire des femmes, qui nous montre que les soins infirmiers sont perçus comme une profession féminine et que le travail des femmes est sous-évalué (Sullivan, 2004).

\section{Autres obstacles à l'influence des soins infirmiers}

D'autres obstacles qui nuisent à l'influence des soins infirmiers dans la société moderne comprennent des demandes extérieures telles que: 1) la structure de financement du système de santé, qui réduit l'influence des infirmières; 2) le fait qu'en tant qu'employées, les infirmières n'ont pas souvent l'occasion de s'exprimer; 3) le pouvoir et le prestige traditionnels des médecins (qui, habituellement, ne sont pas des employés); 4) la perception erronée qu'a le public des soins infirmiers. Les obstacles internes comprennent notre propre culture et nos comportements qui maintiennent en place les relations d'oppression, de même que notre tendance à minimiser nos propres accomplissements (Sullivan, 2004). Tous ces facteurs restreignent la capacité des soins infirmiers à exercer de l'influence, mais aucun d'entre eux ne constitue une entrave insurmontable si nous poursuivons activement notre objectif déclaré de devenir une profession d'influence.

\section{L'influence individuelle}

Maintenant que nous avons examiné certains des obstacles qui empêchent les soins infirmiers d'exercer un rôle influent, peut-être vous demandez-vous pourquoi, en tant qu'individus, vous voudriez avoir de l'influence. L'objectif que nous visons en cherchant à accroître notre influence n'est pas la simple réussite ou le seul gain personnels. L'influence peut servir à améliorer les soins aux patients, à créer de meilleures conditions de travail ou à améliorer l'état de santé de nos concitoyens, pour ne citer que quelques exemples. Vous avez de nombreuses occasions d'exercer de l'influence et il est dommage de gaspiller une occasion de poser un geste constructif. Vous pouvez faire preuve d'influence au chevet, lors de réunions formelles et informelles au travail, dans des comités, dans des conversations de tous les jours, lors de réunions professionnelles au sein de la collectivité, dans des groupes de bénévoles, etc. Les possibilités sont presque illimitées (Sullivan, 2004).

\section{Le jeu}

Une des principales raisons pour lesquelles il est important d'acquérir de l'influence en tant qu'individus est que, même si les femmes ont tendance à ne pas aimer entendre ceci, le travail est un jeu. Que vous le vouliez ou non, le travail d'infirmière est un jeu, et les jeux sont régis par des règles qu'il convient d'apprendre et d'utiliser afin de survivre et de prospérer. L'objectif du travail est la réussite, alors que les objectifs de la vie sociale sont le plaisir et l'approbation. La prospérité passe par la réussite au travail et la capacité de réaliser ses objectifs. Souvent, dans la vie quotidienne, les femmes "gagnent le jeu" en se sentant bien par rapport à ce qu'elles font. Toutefois, les infirmières ont tendance à ne pas mesurer la réussite professionnelle en ces termes, mais plutôt en fonction des récompenses monétaires, de la gratitude et du respect auxquels elles ont droit - trois éléments qui sont trop souvent dispensés au comptegoutte. N'y aurait-il pas dans le domaine des soins infirmiers d'autres objectifs raisonnables dont la réalisation pourrait servir de mesures de la réussite? Des objectifs qui auraient un sens pour nous en tant qu'individus? Par exemple, qu'en serait-il si nous mesurions la réussite en fonction de notre capacité de prodiguer des soins à nos patients, d'enseigner à nos élèves ou de gérer une unité ou un département? Ou en fonction de la satisfaction que nous tirons de notre travail, des nouvelles connaissances que nous acquérons, des nouvelles relations que nous établissons avec des collègues dans le cadre du travail ou avec des personnes qui pourront nous aider à l'avenir (Sullivan, 2004). Si telles étaient les mesures de la réussite, vous sentiriez-vous alors plus accomplies dans le jeu que sont les soins infirmiers? Voilà qui porte à réflexion.

Les jeux ont tous des règles qui nous permettent de jouer et de gagner, mais une des difficultés dans ce jeu est que les règles ne sont écrites nulle part. Il faut pourtant les connaître (et les respecter) si l'on souhaite prospérer. Voici quelques exemples de règles: apprendre à négocier et à échanger des faveurs, reconnaître les jetons du système d'échange, utiliser le bouche-à-oreille pour accumuler cette ressource des plus précieuses qu'est l'information, employer des tactiques non conflictuelles, utiliser notre influence, accepter qu'il se peut que vous deviez perdre une bataille pour gagner la guerre, établir des relations, assumer des responsabilités, devenir une joueuse d'équipe, cultiver la loyauté, chercher des mentors et accepter le risque (Sullivan, 2004).

Il me semble que je peux presque entendre certaines d'entre vous protester: "Et pourquoi devrions-nous suivre les règles? Pourquoi ne pouvons-nous pas faire ce que nous voulons?" Et bien, pour diverses raisons, le non-respect des règles peut certainement vous être nuisible. Il est important de réaliser que les organismes fonctionnent grâce à un système de règles, que les postes hiérarchiques ont du pouvoir et qu'il faut de l'autorité pour assumer des responsabilités. Les symboles de statut sont importants, malgré ce que vous pouvez en penser. Les règles du jeu déterminent le statut, qui à son tour détermine l'assignation des places de stationnement et le droit que s'arrogent certains groupes de fermer la clinique parce qu'ils ont une conférence nationale. L'existence des règles signifie qu'il vous est possible d'influencer la culture organisationnelle. La mise à profit des règles peut vous être avantageuse; ne pas les connaître vous prive d'influence.

Je ne suis certainement pas partisane du respect inconditionnel des règles. Elles demandent parfois d'être enfreintes, mais vous devez savoir quand, où et comment les enfreindre, et ne le faire qu'après avoir soigneusement pesé le pour et le contre. Il est parfois réellement préférable de demander pardon que de demander une permission. Mais il $\mathrm{y}$ a des règles que vous ne devriez jamais enfreindre - ne calomniez jamais votre patron, n'évitez jamais de rembourser une dette, ne sabotez jamais le travail d'une équipe et ne mentez jamais. La violation de ces règles aura certainement des conséquences négatives (Sullivan, 2004).

Bien que les règles du jeu existent, les soins infirmiers ne s'y prêtent pas très bien, pour trois raisons. Premièrement, notre profession est dominée par les femmes, et les règles ont été élaborées par des hommes. Deuxièmement, les soins infirmiers sont une profession où la satisfaction à l'exercer joue un rôle prépondérant, et, pour nombre d'entre nous, l'idée de planifier notre carrière de façon à atteindre la réussite plutôt que d'accepter les félicitations lorsqu'elles se présentent ne semble pas naturelle. Et troisièmement, notre profession a ses propres règles: aucune infirmière n'est plus importante qu'une autre; ne pas tenter de se hisser au-dessus des autres; ne pas se vanter; reconnaître les accomplissements des autres; et n'exclure personne. Vous reconnaissez ces règles? Mais où cela nous mène-t-il? Allons-nous jamais faire des progrès en tant qu'individus et en tant que profession si nous ne disons à personne ce que nous faisons et si nous ne nous félicitons pas de faire du bon travail?

Bien que les règles ne favorisent pas notre profession, vous pouvez tout de même en tirer profit. Cessez de vous comporter comme des victimes et "faites le message à la bonne personne" - autrement dit, cessez de vous plaindre à vos collègues et allez plutôt parler à la personne qui a l'autorité nécessaire pour effectuer un changement (Sullivan, 2004). Défendez vos droits, ceux de vos patients et ceux de votre profession. N'assumez pas de responsabilités si on ne vous donne pas l'autorité nécessaire. Ne faites pas de basses besognes toutes seules. Acceptez le risque. Apprenez à enfreindre les règles aux 
bons moments. Surtout, prenez soin de vous; cela ne nous vient pas naturellement, à nous les infirmières, et pourtant, comme nous l'a rappelé Judy Atkinson lors de sa présentation expérientielle Circles of Rhythm, nous devons être en bonne santé nous-mêmes si nous souhaitons soigner autrui.

\section{L'optimisation de notre capacité d'exercer de l'influence}

Si vous choisissez d'exercer de l'influence, alors vous devez prendre certaines mesures pour maximiser votre capacité dans ce domaine. Notre façon de nous présenter constitue une expression extériorisée de notre expérience intérieure; vous devez donc évaluer les façons dont vous vous présentez. Outre l'apparence, la présentation de soi comprend des comportements subtils et moins subtils et découle de votre attitude envers vous-même. Vous devez évaluer votre capacité actuelle d'exercer une influence. Cette conscience de soi peut naître de l'introspection et de la rétroaction formelle et informelle d'autrui. De plus, vous devez continuellement évaluer vos propres aptitudes en matière d'influence, de façon à pouvoir évaluer l'efficacité de certaines stratégies auprès d'individus et de groupes. L'acquisition d'un rôle influent est un processus qui aiguise la capacité d'écoute et la perspicacité. Cela peut améliorer votre capacité d'être efficaces dans la réalisation de vos objectifs personnels et de ceux de la profession (Sullivan, 2004). Il va sans dire que le processus n'est pas dénué de risques, tels qu'inspirer de l'envie ou de la jalousie chez autrui. Mais ne laissez pas cela vous retenir!

\section{Devenez chef de file}

Maintenant que vous comprenez la façon dont fonctionnent le pouvoir et l'influence, vous devez mettre vos connaissances en pratique en devenant chef de file. Un des énoncés de valeur de l'ACIO affirme que "Chaque infirmière est un chef de file". Nous avons toutes le potentiel de devenir des leaders dans au moins un volet de notre vie, d'avoir une vision de l'avenir et de relever le défi que nous lance Toben lorsqu'elle nous invite à réaliser nos rêves et à devenir nos propres héros. Une chef de file n'a pas besoin de posséder un titre particulier ou un poste d'autorité, et les leaders ne sont pas tous né(e)s ainsi: on peut apprendre à devenir une chef de file. Vous pouvez ajouter à vos qualités personnelles des aptitudes de leadership que vous acquérez dans le cadre de votre formation professionnelle et tout le long de votre carrière. Les infirmières peuvent être des chefs de file au chevet, en gestion, dans la collectivité, dans le milieu universitaire, etc. Les seules limites sont celles que nous nous imposons nous-mêmes. Le leadership requiert de l'innovation et la capacité d'effectuer des changements. Bien que le changement puisse beaucoup déranger certaines personnes, il constitue une partie essentielle de nos vies quotidiennes, tant sur le plan personnel que professionnel (Tamlyn et Reilly, 2003).

Quels sont certains des attributs des leaders en soins infirmiers? Dans notre profession, une chef de file doit nourrir une vision de l'avenir et avoir la capacité de faire de cette vision une réalité. Elle doit également être bien informée de la nature du changement dans la société en général de même qu'au sein du système de santé. Une chef de file doit avoir suffisamment de confiance pour remettre en question le statu quo et promouvoir des solutions de rechange, même si ces solutions s'avèrent parfois impopulaires. Une chef de file doit aussi être visible afin que son travail soit connu et reconnu.

Le leadership requiert avant tout quatre qualités, les quatre piliers du leadership: être informée, faire preuve d'assertivité, assumer ses responsabilités et être capable de défendre une cause. En tant que chef de file, vous devez être consciente de votre état présent et de votre caractère. Quel est votre niveau de maturité, c.-à-d. votre volonté et capacité de vous acquitter d'une tâche? Quel est votre degré de motivation? Vous devez vouloir être une chef de file et être en mesure d'assumer la responsabilité de vos actions et de celles du groupe.
Votre capacité à mener est liée au niveau ou à la base de connaissances que vous avez dans un domaine particulier; vous devez donc posséder de solides connaissances dans les domaines appropriés. Vous devez également être capable de penser de façon claire et stratégique et de comprendre et articuler vos objectifs. Soyez consciente du pouvoir et de la façon dont il s'acquiert et s'exerce, afin de l'utiliser correctement. Connaissez votre propre personnalité ainsi que l'image que les autres ont de vous; p. ex. votre niveau de confiance, d'enthousiasme, de souplesse, de créativité, d'honnêteté, de sincérité, de tact et d'amabilité. L'apparence personnelle est également importante, puisque les leaders doivent être "présentables". Votre vieille tenue de jogging ne convient pas si vous souhaitez que l'on vous prenne au sérieux. Vous devez entretenir de bonnes relations interpersonnelles, d'où le besoin de cultiver vos aptitudes en communication. L'endurance est également nécessaire en raison des demandes stressantes du métier. Il est utile d'avoir un plan personnel de gestion du stress, et vous devez apprendre à dire non et à reconnaitre les moments où il est approprié de dire non. N'oubliez pas que "Non" est une phrase complète! Lorsqu'on vous demande de vous charger d'une tâche que vous n'avez réellement pas le temps de réaliser, cessez de dire "Non, je ne peux pas, mais..." ou "Non, je ne peux pas, mais si tu ne trouves personne d'autre, alors je le ferai". Vous savez ce qui se passe lorsque vous dites cela! Vous devez apprendre à gérer les succès et les échecs et à vous montrer bon joueur dans chaque cas, parce que les échecs sont très probables lorsque vous prenez les risques qui s'imposent pour devenir une bonne chef de file. Paul Brandt nous a rappelé, dimanche, que bien que vous soyez certaines de ne pas échouer si vous ne prenez aucun risque, dans ce cas-là, vous ne vivez pas pleinement votre vie. En dernier lieu, vous devez être capable d'évaluer les forces et les limites de chaque membre du groupe et d'utiliser cette connaissance de façon appropriée, tout en aidant chacun à devenir un membre productif du groupe et à travailler en vue de repousser ses limites et d'atteindre son plein potentiel (Sullivan, 2004). Vous vous sentez épuisées rien qu'à y penser? Ne vous découragez surtout pas! Le jeu en vaut la chandelle!

Une bonne chef de file devrait être assertive sans être agressive. Les personnes qui manquent d'assertivité ont tendance à se rabaisser et à laisser autrui faire des choix et prendre des décisions pour elles, souvent pour éviter le conflit, mais elles finissent souvent par être fâchées contre elles-mêmes et contre autrui. Les personnes agressives sont semblables aux personnes assertives en ce qui a trait aux objectifs, mais elles tentent souvent d'atteindre ces objectifs aux dépens des autres et en ignorant leurs droits. Les chefs de file accomplies savent comment exprimer leurs sentiments, leurs besoins et leurs idées et elles savent défendre leurs droits d'une façon qui tienne aussi compte de ceux d'autrui. Elles protègent leur intégrité de même que celle de l'autre partie. Les personnes assertives établissent des relations étroites, ne permettent pas qu'on se serve d'elles, prennent leurs propres décisions et font leurs propres choix, cernent leurs besoins interpersonnels et y satisfont dans une large part et expriment leurs sentiments positifs et négatifs par des moyens verbaux et non verbaux (Bernhard et Walsh, 1990). Les personnes assertives se sentent généralement bien dans leur peau et ont une opinion positive d'autrui. Pourquoi ne pas vouloir être une personne assertive? En quoi l'assertivité peut-elle vous aider à créer votre avenir en tant qu'infirmières en oncologie? Pensez-y!

La troisième qualité d'une bonne chef de file est la capacité d'assumer ses responsabilités. Ainsi, vous êtes disposée à répondre des conséquences positives ou négatives de vos actes. La responsable, c'est vous. Votre capacité d'assumer vos responsabilités comporte trois volets: premièrement, vous êtes redevable envers vous-même, ce qui signifie que vous devez toujours faire de votre mieux; deuxièmement, vous êtes redevable envers autrui, et cela signifie admettre vos échecs et ne pas fournir d'excuses pour vous protéger; troisièmement, vous avez une responsabilité envers votre profession, 
ce qui signifie que vous devez respecter des normes professionnelles dans le cadre de votre pratique et être disposée à juger vos pairs et à développer votre capacité de former ces jugements.

La capacité de défendre une cause est la dernière qualité requise pour faire une bonne chef de file. Les vraies chefs de file sont capables de soutenir et de défendre la cause de quelqu'un ou de quelque chose. Elles aident un individu ou un groupe à obtenir et à exercer le pouvoir de façon efficace en vue d'effectuer un changement particulier (Bernhard et Walsh, 1990). Une chef de file qui appuie votre cause particulière est un atout précieux que vous devez reconnaître à sa juste valeur. Le fait d'être une chef de file vous aidera à façonner votre avenir ainsi que celui de votre profession.

\section{Joignez-vous à votre organisme professionnel}

Le dernier point que j'aimerais soulever concerne le rôle des organismes professionnels. Comme je vous l'ai dit tout à l'heure, le Canada compte près de 250000 infirmières, et nous représentons presque la moitié des travailleurs de la santé au pays (Construire l'avenir, 2004). Les infirmières sont les travailleurs de la santé les plus respectés du public, et pourtant, nous n'avons pas une voix très manifeste au sein du système de santé. Qu'en serait-il si nous travaillions ensemble - pourrions-nous amener le changement? À mon avis, les organismes professionnels tels que l'ACIO constituent une façon efficace de maximiser l'influence et le pouvoir de la profession, parce qu'ils peuvent parler au nom de leurs membres. L'union fait la force, et n'oubliez pas que pouvoir signifie influence.

Bien que nous n'ayons pas de statistiques claires sur le nombre d'infirmières qui oeuvrent en oncologie à l'échelle du Canada, je soupçonne qu'une bonne proportion des 250000 infirmières du pays travaillent régulièrement avec des patients atteints de cancer. Imaginez le pouvoir et l'influence que pourrait acquérir l'ACIO si nous pouvions réellement parler au nom de milliers d'infirmières! Cependant, l'ACIO ne compte qu'environ 900 membres cette année; c'est beaucoup plus que la plupart des autres spécialités infirmières, mais beaucoup moins que notre potentiel. Des statistiques récentes (Association des infirmières et infirmiers du Canada, 2004) indiquent qu'en 2004, un peu plus de 1200 infirmières ont reçu ou renouvelé leur certification en soins infirmiers en oncologie par le biais de l'Association des infirmières et infirmiers du Canada. Mille deux cents infirmières qui ont fourni un petit effort supplémentaire pour acquérir des connaissances spécialisées, 1200 infirmières qui possèdent les connaissances, le statut, le jargon, etc. leur permettant de communiquer avec les autres membres de l'équipe à un niveau professionnel.

\section{Références}

Bernhard, L., \& Walsh, M. (1990). Leadership: The key to the professionalization of nursing (2nd ed.). Philadelphia, PA: The C.V. Mosby Company.

Construire l'avenir : une stratégie intégrée pour les ressources humaines infirmières au Canada (2004). Soins infirmiers au Canada. Consulté le 20 sept. 2004 à l'adresse :

http://www.buildingthefuture.ca/f/nursing/

Canadian Nurses Association. (2004, June). Section 1 - Update report from the CNA certification program. Ottawa: Author.

McDonald, C. (2003). Issues of gender and power: The significance attributed to nurses' work. In M. McIntyre \& E. Thomlinson (Eds.), Realities of Canadian nursing: Professional, practice, and power issues (pp. 357-371). New York: Lippincott Williams \& Wilkins.
Toutefois, nombre des infirmières certifiées en oncologie à l'échelle du pays ne sont pas membres de l'ACIO, et il convient de se demander pourquoi. Comment pouvons-nous inviter ces infirmières à se joindre à 1 'ACIO et comment pouvons-nous accroître de façon significative notre bassin de membres, étant donné le grand nombre d'infirmières qui travaillent en oncologie, afin que nous puissions acquérir plus d'influence et créer notre avenir nous-mêmes? Vous serez heureuses d'apprendre que le conseil d'administration de l'ACIO se penche diligemment sur ces questions. Mais en attendant, j' aimerais vous demander de réfléchir à ce que vous pouvez faire de vôtre côté - eh bien, certaines d'entre vous ici présentes ne sont pas encore membres de l'ACIO, mais tous les renseignements concernant l'adhésion sont disponibles, il suffit de demander au comptoir d'inscription! Certaines d'entre vous ont des collègues de travail qui ne sont pas membres de l'ACIO. Comment pouvez-vous encourager autrui à devenir membres actifs de l'ACIO afin que nous puissions parler d'une seule voix forte dans le but de diriger la croissance de notre spécialité et l'étendue de la pratique? Il existe de nombreuses possibilités, de la suppression de certains obstacles à l'adhésion à l'obligation d'adhérer à l'ACIO pour les infirmières qui oeuvrent en oncologie. À mon avis, en tant que professionnelles, nous avons l'obligation de contribuer à notre profession de toutes les façons possibles. La participation à votre organisme professionnel est également une façon de stimuler votre vie professionnelle. Imaginez ce qui arriverait si chaque membre de l'ACIO recrutait un seul autre membre en 2005! Imaginez jusqu'où pourraient s'étendre notre pouvoir et notre influence! Il nous serait ainsi beaucoup plus aisé de prendre en main et de créer notre avenir nous-mêmes!

\section{Conclusion}

La fin de ma présentation approche, et j'aimerais maintenant retourner au point de départ et vous poser de nouveau la question: "Les infirmières en oncologie ont-elles le pouvoir de créer leur propre avenir?" Pour ma part, je répondrais "Oui, nous l'avons, mais..." Nous avons le pouvoir, mais nous n'en connaissons pas toujours l'étendue et nous ne savons pas toujours comment l'utiliser de façon avantageuse pour nous. Mon objectif aujourd'hui était de vous sensibiliser davantage aux possibilités qui s'offrent à nous et de vous faire réfléchir sur la façon dont nous pouvons exercer notre pouvoir et notre influence afin de donner une orientation aux soins infirmiers en oncologie. J'espère que vous avez appris quelque chose que vous pourrez emporter avec vous et appliquer dans votre pratique afin de remettre en question le statu quo, d'instituer les changements requis et de créer votre avenir vous-mêmes en tant qu'infirmières en oncologie. Merci.

Roberts, S. (1983). Oppressed group behaviour: Implications for nursing. Advances in Nursing Science, July, 21-30.

Roberts, S. (2000). Development of a positive professional identity: Liberating oneself from the oppressor within. Advances in Nursing Science, 22(4), 71-82.

Ross-Kerr, J. (2002). Gender issues in nursing. In J. Ross-Kerr \& M. Wood (Eds.), Canadian nursing: Issues and perspectives (pp. 83-99). New York: C.V. Mosby Company.

Sullivan, E. (2004). Becoming influential: A guide for nurses. Upper Saddle River, NJ: Pearson Education.

Tamlyn, D., \& Reilly, S. (2003). Innovation and contemporary nursing leadership. In M. McIntyre \& E. Thomlinson (Eds.), Realities of Canadian nursing: Professional, practice, and power issues (pp. 494-515). New York: Lippincott Williams \& Wilkins. 